\title{
ZmCCT regulates photoperiod-dependent flowering and response to stresses in maize
}

\author{
Huihui $\mathrm{Su}^{1 \dagger}$, Jiachen Liang ${ }^{1 \dagger}$, Salah Fatouh Abou-Elwafa ${ }^{2 \dagger}$, Haiyang Cheng ${ }^{1}$, Dandan Dou ${ }^{1}$, Zhenzhen Ren ${ }^{1}$,
} Jiarong Xie ${ }^{1}$, Zhihui Chen ${ }^{1}$, Fengran Gao ${ }^{1}$, Lixia Ku ${ }^{1 *}$ and Yanhui Chen ${ }^{1 *}$

\begin{abstract}
Background: Appropriate flowering time is very important to the success of modern agriculture. Maize (Zea mays L.) is a major cereal crop, originated in tropical areas, with photoperiod sensitivity. Which is an important obstacle to the utilization of tropical/subtropical germplasm resources in temperate regions. However, the study on the regulation mechanism of photoperiod sensitivity of maize is still in the early stage. Although it has been previously reported that $Z m C C T$ is involved in the photoperiod response and delays maize flowering time under long-day conditions, the underlying mechanism remains unclear.

Results: Here, we showed that ZmCCT overexpression delays flowering time and confers maize drought tolerance under LD conditions. Implementing the Gal4-LexA/UAS system identified that ZmCCT has a transcriptional inhibitory activity, while the yeast system showed that ZmCCT has a transcriptional activation activity. DAP-Seq analysis and EMSA indicated that ZmCCT mainly binds to promoters containing the novel motifs CAAAAATC and AAATGGTC. DAP-Seq and RNA-Seq analysis showed that ZmCCT could directly repress the expression of ZmPRR5 and ZmCOL9, and promote the expression of $Z m R V E 6$ to delay flowering under long-day conditions. Moreover, we also demonstrated that $Z m C C T$ directly binds to the promoters of $Z m H Y 5, Z m M P K 3, Z m V O Z 1$ and $Z m A R R 16$ and promotes the expression of $Z m H Y 5$ and $Z m M P K 3$, but represses $Z m V O Z 1$ and $Z m A R R 16$ to enhance stress resistance. Additionally, ZmCCT regulates a set of genes associated with plant development.

Conclusions: $Z m C C T$ has dual functions in regulating maize flowering time and stress response under $L D$ conditions. ZmCCT negatively regulates flowering time and enhances maize drought tolerance under LD conditions. ZmCCT represses most flowering time genes to delay flowering while promotes most stress response genes to enhance stress tolerance. Our data contribute to a comprehensive understanding of the regulatory mechanism of ZmCCT in controlling maize flowering time and stress response.
\end{abstract}

Keywords: DAP-Seq, Maize, Flowering time, Circadian period, Stress response, ZmCCT

*Correspondence: kulixia0371@163.com; chy9890@163.com ${ }^{\dagger} H$ uihui Su, Jiachen Liang and Salah Fatouh Abou-Elwafa contributed equally to this work.

${ }^{1}$ Synergetic Innovation Center of Henan Grain Crops and National Key Laboratory of Wheat and Maize Crop Science, Henan Agricultural University, Zhengzhou 450046, Henan, China

Full list of author information is available at the end of the article

\section{Background}

Maize (Zea mays L.), which was domesticated in Southern Mexico roughly 9000 years ago from Balsas teosinte and spread through North and South America before the arrival of Europeans, requires short-day conditions to flower [1]. Tropical maize genotypes are generally sensitive to long-days condition. Flowering time is one of the most important traits that determines plant adaptability to environmental cues [2,3]. Several genes implicated in maize floral transition have been identified and 
functionally characterized including the CCT domaincontaining genes $Z m C C T, Z m C C T 9$, and the maize CONSTANS LIKE 3 (ZmCOL3), Zea mays MADS-box genes $Z M M 4$ and $Z m M A D S 69$, the circadian clock component genes $Z m C C A 1$ and $Z m C C A 1 a$, Zea mays CENTRORADIALIS 8 (ZCN8), and Zea mays NF-Y transcriptional factor gene $Z m N F-Y A 3$. CCT domain genes play an important role in flowering time regulation in maize [4]. $\mathrm{ZmCOL3}$ appears to affect flowering time primarily under long days (LD) conditions. $\mathrm{ZmCOL3}$ represses flowering time by activating the expression of ZmCCT [4], which functions as a flowering repressor in maize under LD conditions [5, 6]. A Harbinger-like transposable element that tropical SD maize germplasms do not have, acts in cis to promote flowering under LD conditions by repressing the expression of ZmCCT9 [7]. Under LD conditions, $Z m C C T 9$ delays flowering time by negative regulation of the florigen gene $Z C N 8$ [7]. ZCN8 underlies the major flowering time quantitative trait locus $q D T A 8$ that is involved in photoperiod sensitivity $[8,9]$. The MADS-box transcription factors play important roles in flower development and plant inflorescence [10]. $Z m M A D S 69$ suppresses the expression of the flowering repressor $Z m R a p 2.7$, thereby promotes the expression of $Z C N 8$ and causing early flowering [10]. ZMM4 promotes floral transition, and the maize transgenic plants overexpress ZMM4 flowered earlier than the wild type [11]. Plants adjust daily changes and seasonal changes by the circadian clock, an endogenous mechanism that controls a wide range of biological processes [12, 13]. ZmCCA1, an ortholog of AtCCA1, is expressed in a rhythmic pattern, and the overexpression of $Z m C C A 1$ in A. thaliana delayed flowering time [14]. Shi et al. [15] showed that $Z m C C A 1 a$ is likely to be an important component of the circadian clock in maize, and flowering time was delayed in the $Z m C C A 1 a$-overexpressing $A$. thaliana lines under LD conditions. NF-Ys are widespread in eukaryotes. Several studies showed that NF-Ys play an important role in flowering time regulation [16-19]. In Arabidopsis, the $n f$ $y c 3 / y c 4 / y c 9$ triple mutant produced almost twice as many total leaves before flowering compared to the wild type under LD but not SD conditions, indicating that NF-YC3, $N F-Y C 4$ and NF-YC9 genes are involved in photoperioddependent flowering regulation [16]. The overexpression of the $O s N F-Y C 2$ could rescue the late-flowering phenotype of the Arabidopsis $n f-y c 3 / y c 4 / y c 9$ triple mutant [18]. $Z m N F-Y A 3$ encodes an NF-YA subunit in maize, and the zmnf-ya3 mutant showed delayed flowering under LD conditions, whereas there was no significant difference in flowering time compared to the WT under SD conditions [19].

A growing number of evidence showed that photoperiod responsive genes play important roles in plant response to biotic and abiotic stresses [20-23]. Tian et al. [20] showed that transgenic Arabidopsis plants overexpressing $Z m C C A 1.1$ exhibited higher tolerance to drought stress. AtPRR7, a central component of the Arabidopsis clock, negatively regulates stress responses via direct regulation of drought- and abscisic acidresponsive genes [21]. Wang et al. [22] identified new genes responsible for $R$-gene-mediated resistance to downy mildew in Arabidopsis, and they are controlled by the circadian rhythm regulator CCA1. Abscisic acid, osmotic and salt stresses could induce the expression of AtCOL4 [23], which is an essential regulator of abiotic stress tolerance in Arabidopsis. Su et al. [19] showed that $Z m N F-Y A 3$ enhances stress resistance in maize under LD conditions rather than positive regulation of flowering time. The relative water contents (RWC) of zmnf-ya3 mutant was significantly lower than WT plants after 1 and 4 days of drought and heat stress induction under LD conditions, respectively. ZmNF-YA3 improves plant tolerance to drought and heat stresses via direct regulation of the expression of the bHLH92, FAM and JA activator gene MYC4 [19].

A previous study revealed that $Z m C C T$ plays a negative role in regulating flowering time in maize $[5,6]$. Hung et al. [5] showed that the expression of $Z m C C T$ alleles from diverse teosintes is higher than that from temperate maize and confer delayed flowering phenotype under LD conditions. In early-flowering maize, Yang et al. [6] detected a CACTA-like transposable element within the $Z m C C T$ promoter that markedly reduces flowering time. The CACTA-like transposable element represses the expression of $Z m C C T$ to reduce photoperiod sensitivity. Consequently, maize could flower early under LD conditions. $Z m C C T$ is a homolog of the rice photoperiod response regulator gene Ghd7. Ghd7 is diurnally expressed, and the expression of Ghd7 was much higher under LD compared to SD conditions. Ghd7 is expressed in young tissues, such as developing leaves, apical meristem and leaf sheaths [24]. The teosinte $Z m C C T$ allele showed a diurnal expression pattern under LD conditions, with higher transcription levels observed in the light [6]. ZmCCT is expressed in the leaves and shoot apical meristem at 3-7 leaf stages, plant ovule and pollen [25-27]. Enhanced expression of Ghd7 under LD conditions delays heading date in rice [24]. The expressions of $H d 3 a$ and Ehd 1 were suppressed by Ghd7 under LD conditions [24]. Ku et al. [25] showed that the RWC of the HZ4-NIL containing ZmCCT-associated QTL was remarkably higher than that of HZ4 after drought and heat stress induction. The co-expression analysis and the diurnal rhythms of stress response-related genes suggest $Z m C C T$ as a crucial functional crosslink linking photoperiod with stress tolerance responses under LD 
conditions [25]. However, the molecular mechanisms of $\mathrm{ZmCCT}$ in photoperiod-dependent flowering time regulation and response to biotic/abiotic stresses in maize are still ambiguous. Here we showed that overexpression of $\mathrm{ZmCCT}$ delays flowering time and confers drought tolerance in maize under LD conditions. Implementing the Gal4-LexA/UAS system revealed that $Z m C C T$ has a transcriptional inhibitory activity, while the yeast system showed that $Z m C C T$ has a transcriptional activation activity. The DAP-Seq assay and EMSA showed that $Z m C C T$ binds to the promoters containing the motifs CAAAAATC and AAATGGTC. Moreover, DAP-Seq and RNA-Seq analyses showed that $Z m C C T$ regulates genes implicated in photoperiod-dependent flowering time regulation, stress response and plant development. This work contributes to a comprehensive understanding of the molecular mechanism of photoperiod affecting maize flowering time and stress response.

\section{Results}

Phenotypic variations in flowering time and drought stress tolerance under LD conditions

Seven $\mathrm{T}_{0}$ transgenic plants were obtained from three independent transgenic events. Compared to the WT plants, $\mathrm{T}_{2}$ families of transgenic plants exhibited 5.9 days delayed anthesis under LD conditions (Fig. 1a, b). Under SD conditions, no significant differences were observed between OE- ZmCCT and the WT plants in the number of days to anthesis (Fig. 1a, b). The total leaf number (TLN) of the OE-ZmCCT was higher than WT plants under LD conditions (Fig. 1c). These results suggest that overexpression of $Z m C C T$ delays maize flowering under LD conditions. Moreover, the OE-ZmCCT and WT plants exhibited significant differences in response to drought stress under LD conditions (Fig. 1d). The relative water content (RWC) was estimated to investigate the response of the OE-ZmCCT and WT plants to
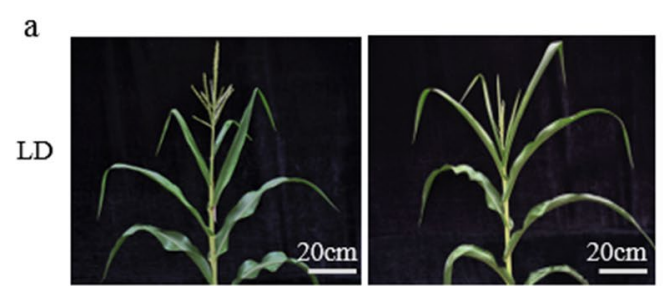

b

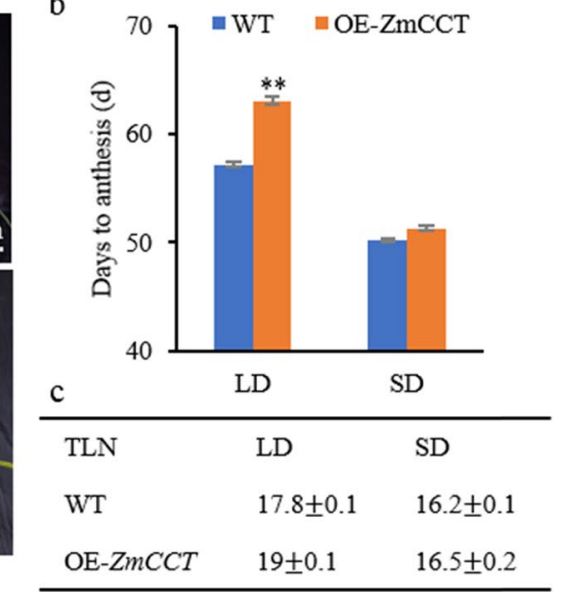

d
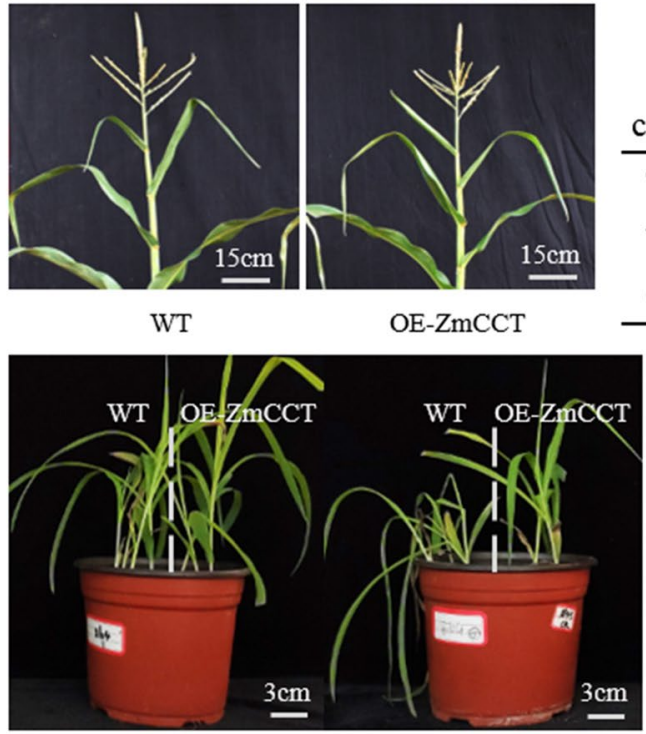

WI

DS

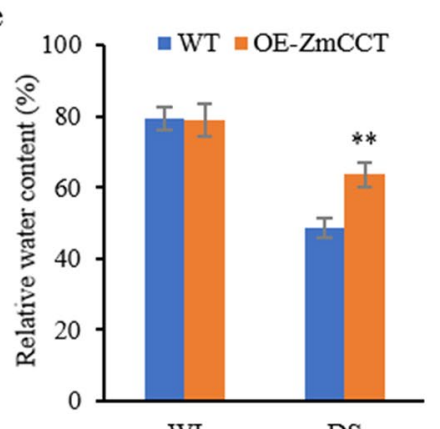

WI

DS

Fig. 1 Phenotypic evaluation of flowering time and stress response of ZmCCT. a) Phenotypes of the WT and OE-ZmCCT lines under LD and SD conditions at the $V 6$ growth stage. Bar $=15$ or $20 \mathrm{~cm}$. b) Flowering time of the WT and OE-ZmCCT lines under SD and LD conditions. Seven $T_{2}$ transgenic lines were evaluated. Data are shown as an average $(n=3, \pm S D, * * P<0.01)$. c) The total leaf number (TLN) of the WT and OE-ZmCCT lines under LD and SD conditions. Sixteen plants were used from each line. Data are shown as an average \pm SD. d) Phenotypes of the WT and OE- $Z m C C T$ lines under well-irrigated (WI) and drought-stressed (DS) treatments under LD conditions. Bar $=3 \mathrm{~cm}$. e) Relative water content (RWC) of the WT and OE-ZmCCT lines under well-irrigated (WI) and drought-stressed (DS) treatments. Two $\mathrm{T}_{2}$ transgenic lines were used. (Data are shown as an average $\left.(n=3) \pm S D,{ }^{* *} P<0.01\right)$ 
drought stress at the physiological level. The RWC of OE$Z m C C T$ plants was significantly higher than that of the WT plants after drought stress induction (Fig. 1e). These results indicate that $\mathrm{ZmCCT}$ has dual functions in regulating maize flowering time and stress response under LD conditions.

\section{RNA-Seq identification of genes affected by $Z m C C T$}

To understand the regulatory network of ZmCCT in response to photoperiod-mediated flowering, RNA-Seq analysis was conducted using total RNA extracted from leaves of the wild type (WT) and ZmCCT over-expressing plants $(\mathrm{OE}-\mathrm{Z} m C C T)$ at $\mathrm{V} 6$ growth stage under both SD and LD conditions. On average, 21.4-27.3 million 150-nt clean reads were generated for each cDNA library (12 cDNA libraries in total), and $~ 83 \%$ of the reads were uniquely mapped to the maize reference genome V4 (Additional file 1). To dissect the changes in the gene expression in response to LD conditions, differentially expressed genes (DEGs) between the WT and OE$Z m C C T$ plants under SD and LD conditions were investigated. Genes showed significant changes in expression (> two-fold, i.e., $\log 2$ foldchange $>1$ or $\log 2$ foldchange $<-1$, padj $<0.05)$ were selected for further analysis. Accordingly, 91 and 746 genes DEGs were identified under SD and LD conditions, respectively (Fig. 2a). To validate the differential expression observed from RNA-Seq analysis between the WT and OE-ZmCCT under SD and LD conditions, RT-qPCR on 20 DEGs under LD conditions was performed (Additional file 2). RT-qPCR of those 20 DEGs showed similar levels of differential expression patterns observed from the RNA-Seq analysis, indicating

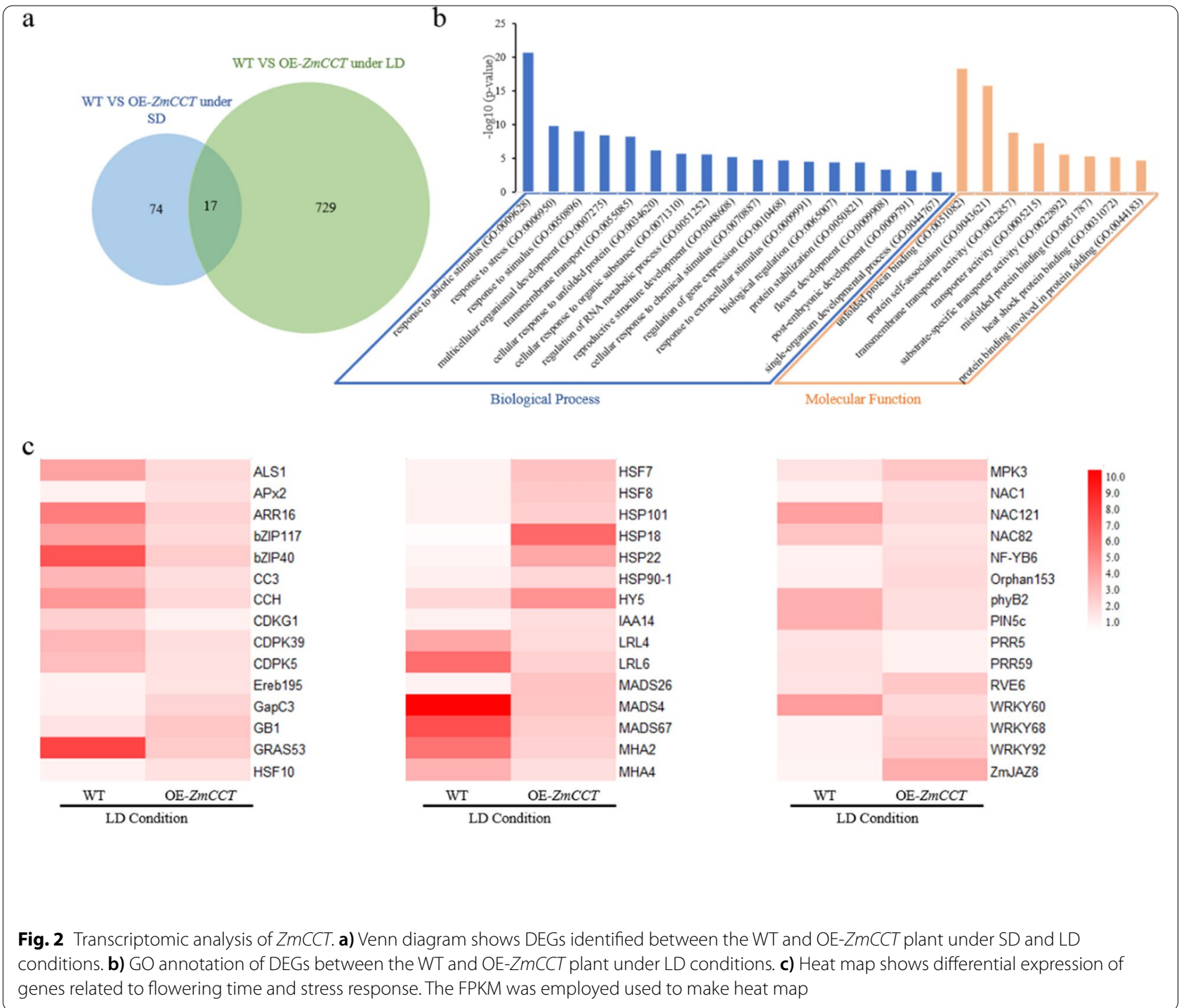


the reliability of RNA-Seq analysis in the identification of DEGs (Additional file 3, Fig. S1). The agriGO v2.0 analysis toolkit was employed to perform Gene Ontology (GO) enrichment analysis [28]. Out of the 746 DEGs identified between the WT and OE-ZmCCT under LD conditions, 599 DEGs were functionally annotated (Fig. 2a, b). The GO term enrichment analysis of the 599 DEGs revealed that these genes are mainly involved in cellular process (GO:0050794, $p$ value $\left.=5.2 \times 10^{-4}\right)$, response to stimulus (GO:0050896, $p$ value $\left.=8.00 \times 10^{-10}\right)$, metabolic process (GO:0019219, $p$ value $=1.2 \times 10^{-6}$ ), biological regulation (GO:0065007, $p$ value $=3.4 \times 10^{-5}$ ), developmental process (GO:0044767, $p$ value $=0.001$ ), flower development (GO:0009908, $p$ value $\left.=4.1 \times 10^{-4}\right)$. The most significant subcategory was "response to abiotic stimulus" (GO:0009628, $p$ value $\left.=2.00 \times 10^{-21}\right)$. The other interesting significant subcategories are "response to stimulus" and "flower development". Heatmap showed some differentially expressed genes between the $\mathrm{WT}$ and OE-ZmCCT under LD conditions (Fig. 2c, Additional file 4).

\section{ZmCCT has both transcriptional activation and inhibitory activities}

To investigate whether $Z m C C T$ can act as a gene expression promotor or repressor, transactivation analysis of $Z m C C T$ in yeast was performed. $Z m C C T$ exhibits obvious transcriptional activation activity (Fig. 3a). However, $\mathrm{ZmCCT}$ encodes a CCT-domain protein (Fig. $3 \mathrm{~b}$ ) that has been reported to have a transcriptional inhibitory activity [21, 29]. To confirm that, the Gal4-LexA/UAS system that analyzes the proteins for positive or negative transcriptional potential was implemented. $Z m C C T$ significantly decreased the expression of the reporter gene (Fig. 3c), indicating that $Z m C C T$ has a transcriptional inhibitory activity. Altogether, $Z m C C T$ is likely to have both transcriptional activation and inhibitory activities.

\section{DAP-Seq identifies $Z m C C T$ target genes}

DAP-Seq assay was employed to identify the potential target genes directly regulated by $Z m C C T$. Using the Illumina platform (PE150 sequencing strategy), the DAP-Seq assay produced about 30 million reads of two biological

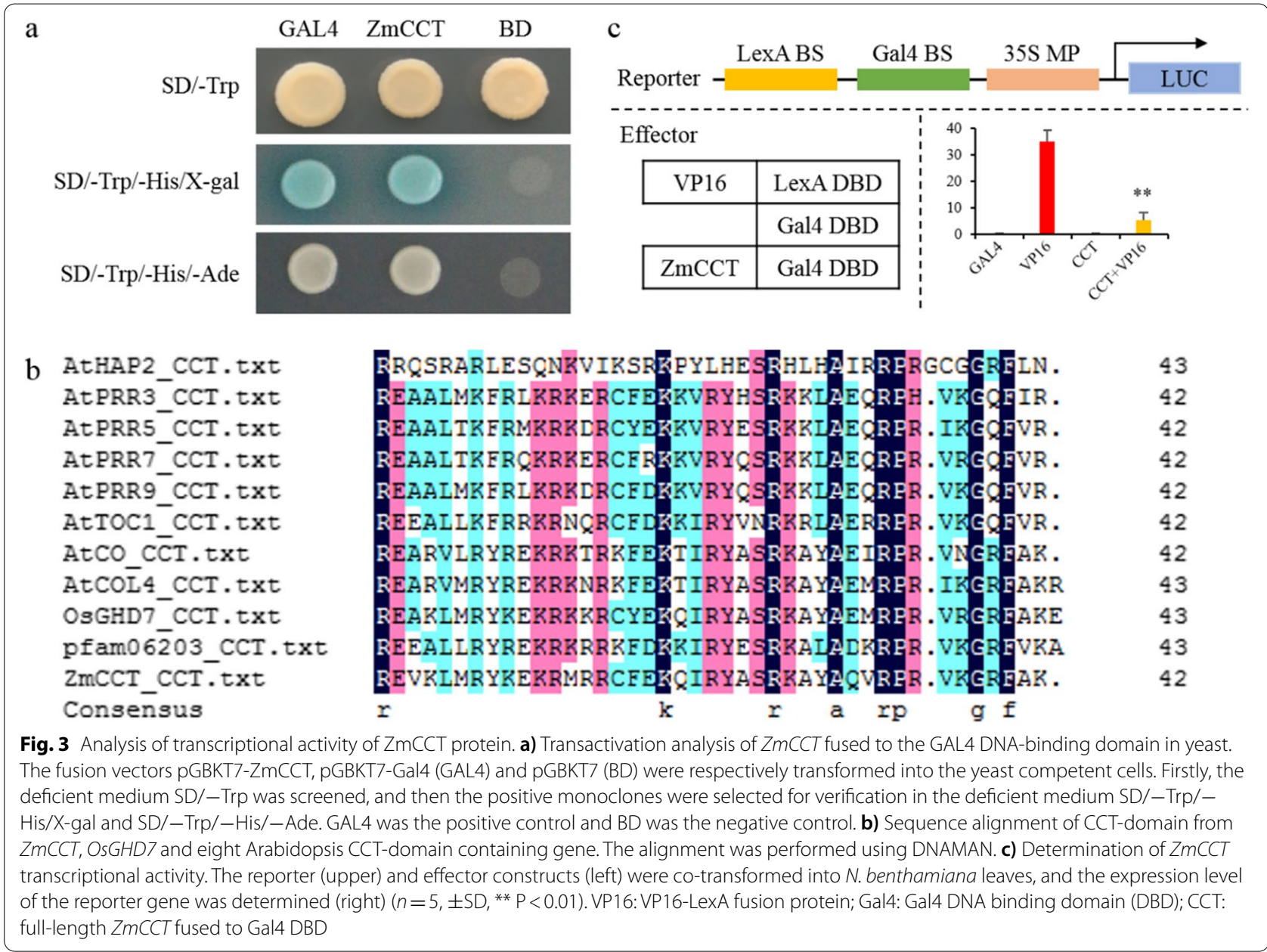


replicates. Out of those reads, about 21 million reads were uniquely mapped to the maize_V4 reference genome. The effective mapping ratio was about $72 \%$. The MACS2 algorithm version 2.2.7.1 was employed to identify $\mathrm{ZmCCT}$ binding sites. A total of 15,862 peaks across the entire maize V4 genome in the two biological replicates are significantly associated with two motifs, i.e., AAATGG TC and CAAAAATC (Fig. 4a) ( $p$-value <0.05). From all detected peaks, about 21\% (3133 peaks) were located to genic regions including the promoter $(-3 \mathrm{~kb}$ to TSS), 5'UTR, 3'UTR, intron and exon (TTS to $3 \mathrm{~kb}$ ) (Fig. 4b). The 3133 peaks correspond to 2041 genes. GO enrichment analysis revealed that these genes are significantly enriched in the response to stimulus and flower development subcategories (Fig. 4c). Out of 2041 genes, $\mathrm{ZmCCT}$ is likely to bind to the promoter region of 1602 genes, and these genes are mainly involved in the cellular process (GO:0044763, $p$ value $=5.1 \times 10^{-4}$ ), organelle organization (GO:0006996, $p$ value $=4.5 \times 10^{-4}$ ), flower development (GO:0009908, $p$ value $=6.2 \times 10^{-4}$ ), transcription factor activity (GO:0000995, $p$ value $=4.5 \times 10^{-4}$ ) and response to stimulus subcategories (GO:0050896, $p$ value $=2.1 \times 10^{-6}$ ).

To further validate the expression level of those genes as putative targets directly regulated by $Z m C C T$, we analyzed the overlapping genes between DEGs generated from the WT and OE-ZmCCT under LD conditions and putative target genes directly regulated by $Z m C C T$ in the upstream regions. Twenty-one DEGs that are likely to be implicated in flowering time regulation, stress response and flower development were identified (Additional file 5). Out of those 21 genes, 11 genes were upregulated in the $\mathrm{OE}-\mathrm{ZmCCT}$ plants, whereas the remaining 10 genes were downregulated.

\section{Binding motif analysis revealed novel ZmCCT cis-elements} The two novel DNA motifs AAATGGTC and CAAAAATC were selected as candidate binding sites by MEME-ChIP software (Fig. 4a). To confirm whether $Z m C C T$ could bind to the AAATGGTC and CAAAAATC motifs, the electrophoretic mobility shift assay (EMSA) was performed using a purified recombinant $\mathrm{ZmCCT}$ protein and labeled DNA probes containing the $Z m C C T$ binding sites, i.e., AAA TGGTC and CAAAAATC. As shown in Fig. 4d, ZmCCT protein could bind to the AAATGGTC and CAAAAA TC motifs. The addition of $100 \times$ unlabeled competitors reduced the detected binding sites of $Z m C C T$, and it could not bind to the mutated probes (ACGCTAGA and TCC GCGCT). In the absence of the $\mathrm{ZmCCT}$ protein, except for the free probe, no binding band was observed. These results confirmed the specific binding of the $\mathrm{ZmCCT}$ protein to the AAATGGTC and CAAAAATC motifs.

\section{ZmCCT directly regulates genes related to photoperiod-dependent flowering time}

The DAP-Seq and RNA-Seq analyses identified 10 potential target genes of $\mathrm{ZmCCT}$ related to flowering regulation (Additional file 6), from which two PSEUDO RESPONSE REGULATOR (ZmPRR5), a REVEILLE 6 (ZmRVE6) and a CONSTANS-LIKE ( $Z m C O L 9)$. To investigate whether $Z m C C T$ could regulate the expression of these potential target genes, a dual-luciferase transient transcriptional activity assay (referred to as Dual-LUC hereafter) in $N$. benthamiana leaves was carried out. The 35S::ZmCCT served as an effector and $L U C$ (the firefly luciferase-coding gene) driven by different promoter regions $(-3000$ to $-100 \mathrm{bp}$ ) of the potential target genes as a reporter (Fig. 5a). The results indicated that $\mathrm{ZmCCT}$ protein promotes the expression of three of the ten target genes, while represses the expression of the remaining seven genes (Fig. 5b). In particular, $Z m C C T$ binds to the promoters of ZmPRR5 (-1567bp), ZmRVE6 (-420bp) and ZmCOL9 $(-1315 \mathrm{bp})$ to promotes the expression of $Z m R V E 6$, and represses the expression of $Z m P R R 5$ and $Z m C O L 9$ (Fig. 5b, Additional file 3, Fig. S2a).

\section{$Z m C C T$ directly regulates a suite of stress-response genes}

RNA-Seq exhibited differential expression of nine genes associated with the plant response to biotic and abiotic stresses (Additional file 7),, from which six genes were upregulated and three were downregulated in the $\mathrm{OE}$ $Z m C C T$. The Dual-LUC assay results confirmed that of the RNA-Seq (Fig. 5b, 6a, Additional file 7). Three of those nine genes, i.e., ZmVOZ1, ZmARR16, ZmHY5, and ZmMPK3, are known to be involved in drought stress response [30-33]. The results further showed that ZmCCT binds to the promoters of $Z m V O Z 1(-927 \mathrm{bp})$ and $Z m A R R 16$ $(-2438 \mathrm{bp})$ to repress their expression, and to the promoters of $Z m H Y 5$ ( $-2940 \mathrm{bp})$ and $Z m M P K 3(-2841 \mathrm{bp})$ to promote their expression (Fig. 5b, 6a, Additional file 3, Fig. S2b). To further confirm that, we measured the mRNA levels of these nine genes by RT-qPCR in the WT and OE$Z m C C T$ plants between the well-irrigated and droughtstressed treatments under LD conditions. RT-qPCR results showed that the expression of $Z m H Y 5$ and $Z m M P K 3$ was upregulated in the OE-ZmCCT and WT plants in response to drought stress, with the expression levels were much

(See figure on next page.)

Fig. 4 DAP-Seq analysis of ZmCCT target genes. a) ZmCCT binds to the AAATGGTC and CAAAAATC motifs as identified by the MEME-ChIP. b) Distribution of the ZmCCT binding sites in the maize V4 genome. c) GO annotation of targeted genes bound by ZmCCT protein. The $y$-axis represents the percentage of genes related to each functional category. d) Results of EMSAs confirming the ZmCCT binding to the AAATGGTC and CAAAAATC motifs 
a

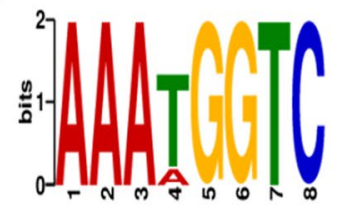

$\mathrm{E}$-Value $=1.1 \mathrm{e}-167$

$\mathrm{c}$

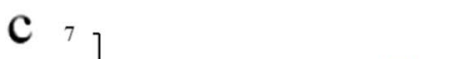

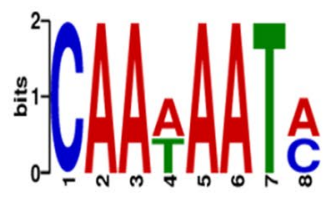

$\mathrm{E}-$ Value $=1.5 \mathrm{e}-81$
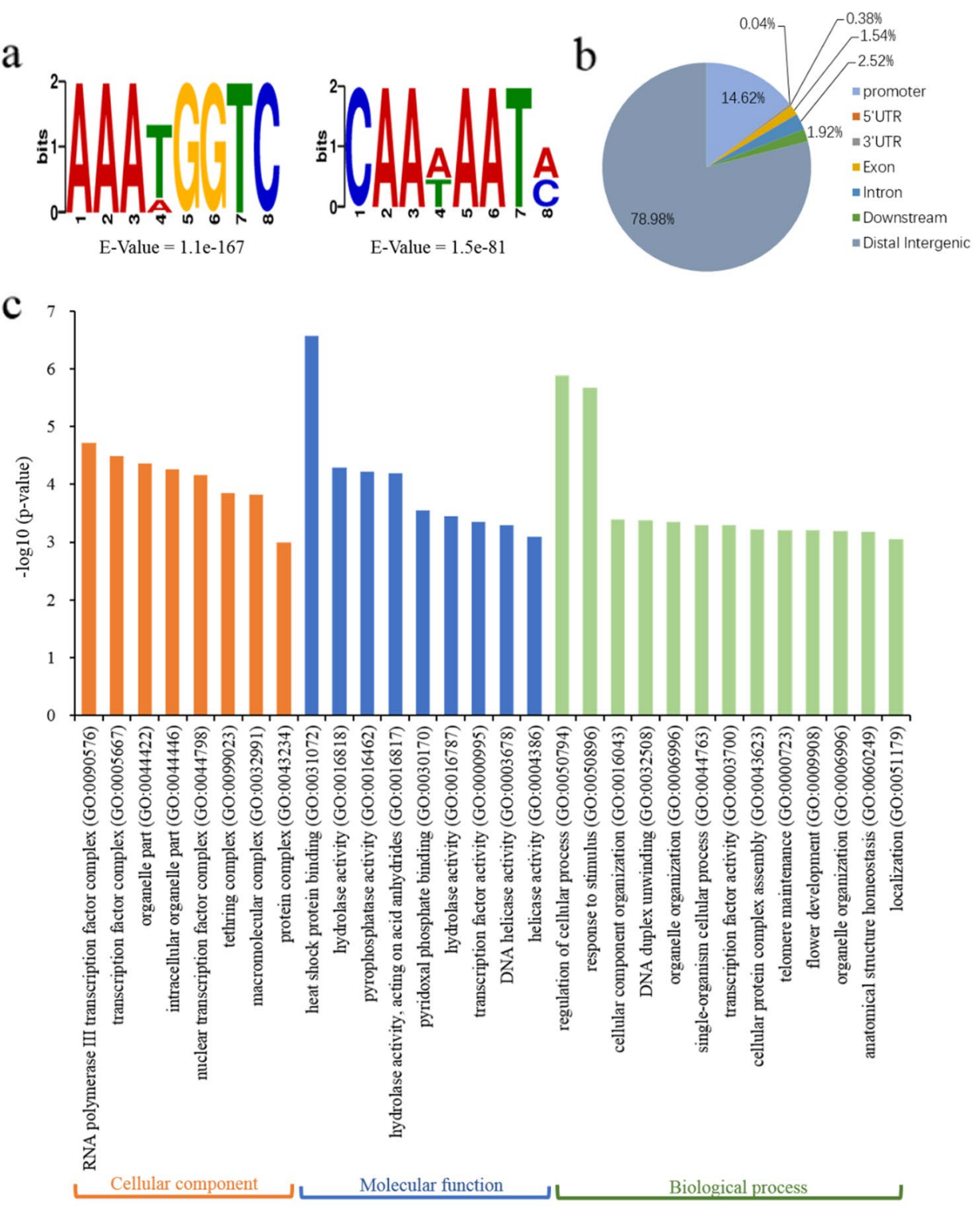

d

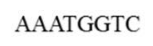

CAAAAATC

Probe

Competitor

ZmCCT

Mutant probe

Complex $\rightarrow$
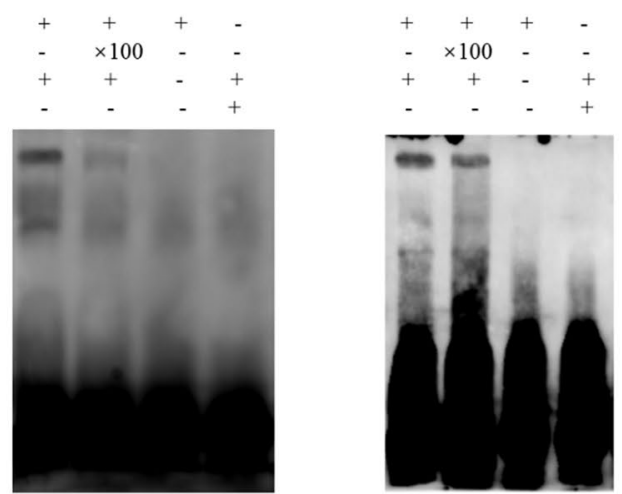

Fig. 4 (See legend on previous page.) 

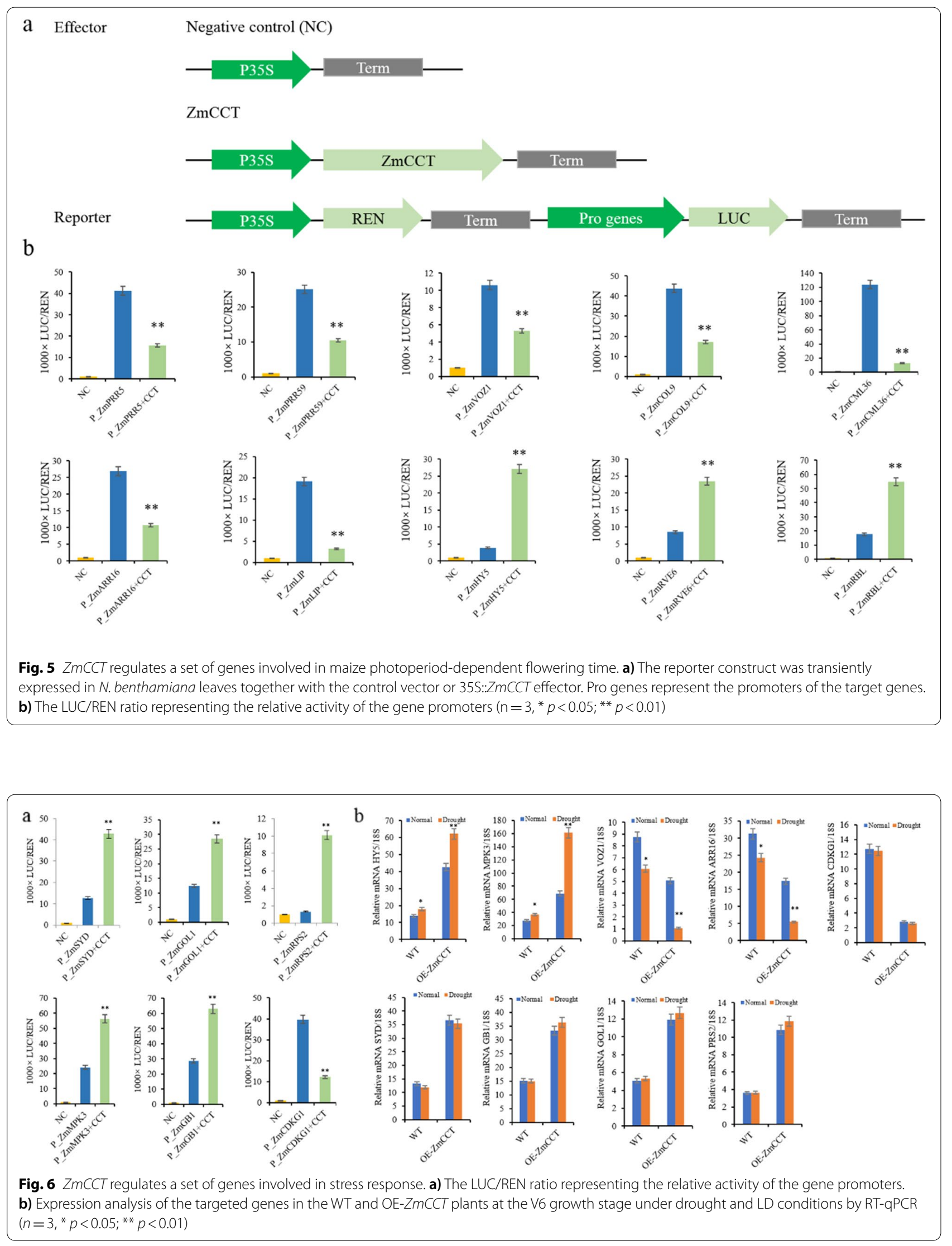
higher in the OE-ZmCCT compared to the WT plants (Fig. 6b). Meanwhile, $Z m V O Z 1$ and $Z m A R R 16$ were downregulated in the $\mathrm{OE}-\mathrm{ZmCCT}$ and WT plants after drought stress induction, and the expression levels were much lower in the OE-ZmCCT compared to the WT plants (Fig. 6b). The expression levels of the remaining 5 genes did not reveal any significant difference between the well-irrigated and the drought-stressed treatments (Fig. 6b). These results indicate that $Z m C C T$ responds to drought stress primarily by direct regulation of the expression of those four drought-stress-related genes (ZmHY5, ZmMPK3, $Z m V O Z 1$ and $Z m A R R 16)$.

\section{ZmCCT directly regulates several development-related genes}

Six DEGs related to the developmental process were identified as direct targets of $\mathrm{ZmCCT}$ (Additional file 5). Four genes were upregulated and two genes were downregulated in the OE- $Z m C C T$ plants. DAP-Seq results showed that $Z m C C T$ has binding sites to the promoters of Zm00001d018977 (ZmMET1, - 1223bp), Zm00001d037982 (FAC, - 2850bp), Zm00001d034929 (ZmLUG3, - 2995bp), Zm00001d044815 (BOB1, - $972 \mathrm{bp}), \mathrm{Zm00001d008882}(\mathrm{ZmM} 2,-2958 \mathrm{bp})$, and $Z m G B 1$ ( $-1587 \mathrm{bp}$ ) (Additional file 5). These genes are involved in regulating plant growth and development.

\section{Discussion}

Flowering time is an important agronomic trait that determines plant adaptation and distribution. The CCT domain-containing gene $Z m C C T$ has been previously reported to play a negative role in regulating flowering time in maize [5, 6]. $Z m C C T$ is a homolog to the rice photoperiod response regulator gene Ghd7. Enhanced expression levels of Ghd7 under LD conditions delay heading date in rice [24]. Here we have employed several molecular techniques including DAP-Seq and RNASeq analyses to elaborate the molecular mechanisms of $\mathrm{ZmCCT}$ in photoperiod-dependent flowering time regulation and response to biotic/abiotic stresses in maize. Our data showed that $Z m C C T$ negatively regulates maize flowering time and confers maize drought tolerance under LD conditions. The observed transcriptional activation and inhibitory activities displayed by $Z m C C T$ when employing either the yeast and Gal4-LexA/UAS transcriptional systems might be due to the interaction with different transcription factors as the Yes-associated protein (YAP) and PDZ-binding motif (TAZ) do, which are known as oncogenic transcriptional co-activators and key regulators of stem cell function [34, 35]. Kim et al. [36] showed that YAP and TAZ could also function as transcriptional co-repressors when interacting with the TEA domain (TEAD) transcription factor. However, further studies are needed to explore the molecular mechanism of $Z m C C T$.

\section{$Z m C C T$ delays flowering by regulating photoperiod-dependent flowering genes}

The observed delayed flowering time in response to the overexpression of $Z m C C T$ under LD conditions is consistent with previous studies $[5,6]$ where $Z m C C T$ , a homolog of the rice photoperiod response regulator $G h d 7$, was consistently expressed at higher levels and confer delayed flowering in the teosintes under LD conditions. The DAP-Seq and RNA-Seq analyses results showed that $Z m C C T$ directly promotes the expression of $Z m R V E 6$, while represses the expression of $Z m P R R 5$ and $Z m C O L 9$, resulted in a delayed flowering phenotype in maize. These findings suggest that $Z m C C T$ delays flowering by upregulating the expression of a rhythmic gene $Z m R V E 6$ and downregulating the expression of the circadian oscillator gene $Z m P R R 5$ and a photoperiodsensitivity gene $Z m C O L 9$. These three genes (ZmRVE6, $Z m P R R 5$ and $Z m C O L 9)$ are involved in the photoperiod-dependent flowering time regulation and circadian rhythm in maize [37-40]. ZmPRR5, which is related to the domestication of maize, reduces the expression levels in the late-flowering phenotype $l f y 1$ mutant compared to the WT [37, 38]. ZmRVE6 is a homolog of the Arabidopsis REVEILLE 8 (AtRVE8) that is involved in the photoperiodic flowering of Arabidopsis and shows a rhythmic expression in maize [39, 40]. Moreover, RVE8 induced several evening-phased oscillator genes in Arabidopsis, including PRR5 [41]. ZmCOL9 belongs to C2C2-COlike-transcription factor 5 and participates in the photoperiod-dependent teosinte flowering pathway [42, 43]. $\mathrm{ZmCOL9}$ is a homolog of the negative regulator of flowering in the photoperiod pathway in Arabidopsis AtCOL9 [44], and $D T H 2$ that acts as an activator of rice heading under long-day conditions [45].

Moreover, RNA-Seq analysis revealed 10 DEGs between the WT and OE-ZmCCT plants under LD conditions. These genes belong to the circadian rhythm, photoperiodism regulation and flower development (Additional file 8), and comprise 3 CCT-domain-containing protein, 3 MADS transcription factor, Adagio1, PHOT2, phyB2 and GRAS53. The expression levels of seven out of those ten genes are downregulated in the OE-ZmCCT plants. The circadian clock is an endogenous mechanism for keeping time, which allows organisms to coordinate biological processes and provide an adaptive advantage $[46,47]$. our data showed that $Z m C C T$ regulates the expression of ZmHY5, ZmARR16, ZmCML36 and $Z m L I P$, which are related to the maintenance of the 
circadian period. More specifically, $Z m C C T$ promotes the expression of $Z m H Y 5$ but represses the expression of $Z m A R R 16, Z m C M L 36$ and ZmLIP under LD conditions, which may prolong the circadian period thus delay flowering time. Under the blue light, a mutation in the HYS in Arabidopsis remarkably shortens the circadian period [48]. AtHY5 affects the clock via the transcriptional repression of PRR5 [48]. Homologs of the ZmARR16 in Arabidopsis, i.e., $A R R 3$ and $A R R 4$, are key genes for a proper circadian period and define an additional level of the circadian clock regulation [49]. ARR3 or ARR4 mutant could prolong the period of the clock regardless of the presence or absence of light [49]. In Arabidopsis, the cytosolic-free $\mathrm{Ca}^{2+}\left(\left[\mathrm{Ca}^{2+}\right] \mathrm{cyt}\right)$ circadian oscillations could influence the function of the circadian clock via the $\mathrm{Ca}^{2+-}$ dependent action of CALMODULIN-LIKE24 (CML24) [50]. The cml23-2cml24-4 double mutant prolongs the circadian period [50]. The Arabidopsis homolog of the $Z m L I P(A t L I P 1)$ acts as a unique negative regulator in controlling the light input of the circadian clock, which is necessary for precise clock entrainment in plants. The lip 1-1 mutant shortens the circadian period by $1.5-2 \mathrm{~h}$ than the wild-type plants under continuous red light in Arabidopsis [51].

Taken together, our results suggest that $Z m C C T$ could directly or indirectly regulate the expression of several photoperiod-dependent and circadian clock maintenance flowering time genes to affect maize flowering time.

\section{$Z m C C T$ improves stress tolerance by regulating a set of stress response genes}

Among the target genes of $\mathrm{ZmCCT}$, several genes were shown to be associated with plant response to various biotic and abiotic stresses, which is consistent with a previous study where $Z m C C T$ has been reported to be implicated not only in regulating flowering time but also in the stress response [25]. In particular, the overexpression of $Z m C C T$ confers maize drought tolerance under LD conditions by regulating the expression of drought stressresponsive genes. After drought stress induction, $\mathrm{ZmHY5}$ and $Z m M P K 3$ expression in transgenic plants overexpressing the $Z m C C T$ transgene was strongly elevated compared to the WT, suggesting that the effect of $Z m H Y 5$ and $Z m M P K 3$ on drought stress is mediated through the promotion of $Z m C C T$. These results are consistent with previous studies where drought stress promotes the expression of $Z m H Y 5$ and $Z m M P K 3$ [30, 31]. Similarly, the strong downregulation of the drought-responsive genes $Z m V O Z 1$ and $Z m A R R 16$ in the OE-ZmCCT plants compared to the WT after drought induction suggests that $Z m C C T$ mediates drought tolerance by suppressing the expression of $Z m V O Z 1$ and $Z m A R R 16$. A previous study showed that $Z m A R R 16$ was downregulated in the ear, leaf and kernel of maize under drought stress [32]. The voz1voz2 double mutant enhanced drought-stress tolerance in Arabidopsis [33]. Furthermore, $\mathrm{ZmCCT}$ may have a role in response to biotic stresses by regulating target genes. For instance, $\mathrm{ZmVOZ1}$ was downregulated in response to the infection with the rice black-streaked dwarf virus (RBSDV) [52]. Moreover, the expression of $Z m M P K 3$ was elevated in response to the infection with Setosphaeria turcica and necrotrophic fungi Cercospora zeae-maydis and Cercospora Zeina [53, 54]. Besides, the strong promoted expression of the defense and immune responsive genes $Z m S Y D, Z m G B 1$ and $Z m R P S 2$ [55-59] in the transgenic plants carrying the $Z m C C T$ transgene compared with WT suggests that $Z m C C T$ plays a crucial role in maize response to biotic stresses.

ZmCCT might be involved in regulating plant development The identification of several $\mathrm{ZmCCT}$ target genes implicated in regulating plant growth and development, such as ZmGB1, ZmLUG3 and ZmM2 suggests that $Z m C C T$ plays indirect roles in regulating plant development. $Z m G B 1$ encodes a heterotrimeric $G$ protein $\beta$ subunit and regulates shoot meristem development in maize [60]. ZmLUG3 protein belongs to the Gro/Tup1 family, which acts as negative transcriptional regulators and play important roles in the growth and developmental processes of many organisms [61]. ZmM2 is involved in maize ear development and is regulated by the BRANCHED SILKLESS 1 (BD1) [62]. The mRNA of $Z m M 2$ was not detected during the development of $b d 1$ ears [62]. Besides, $Z m C C T$ regulates the expression of the Arabidopsis and rice homologs $Z m F A C$, and $Z m M E T 1$ which are known to be involved in plant development [6365]. ZmCCT may be involved in plant growth and development by regulating those development related genes.

Accordingly, we proposed a regulatory model for the role of $Z m C C T$ in regulating flowering time and stress response in maize (Fig. 7). Previous studies on the model plant Arabidopsis revealed that ZmPRR5 and ZmRVE6 may form a negative feedback loop in maize [40, 41]. Interestingly, three genes, i.e., $\mathrm{ZmHY5}, \mathrm{ZmVOZ1}$ and $Z m A R R 16$, might have dual functions in regulating flowering time and stress response [30, 32, 33, 48, 49, 66]. Our data showed $\mathrm{d}$ that $\mathrm{ZmCCT}$ represses the expression of most flowering time genes, whereas promotes the expression of most stress-responsive genes. Two lines of evidence suggest that $Z m C C T$ delays flowering by repressing flowering time-related genes and enhances stress tolerance by promoting the stress-responsive genes. Firstly, the RNA-Seq results showed a strong downregulation of seven of the ten DEGs related to flowering time in the transgenic plants overexpressing the $Z m C C T$ transgene 


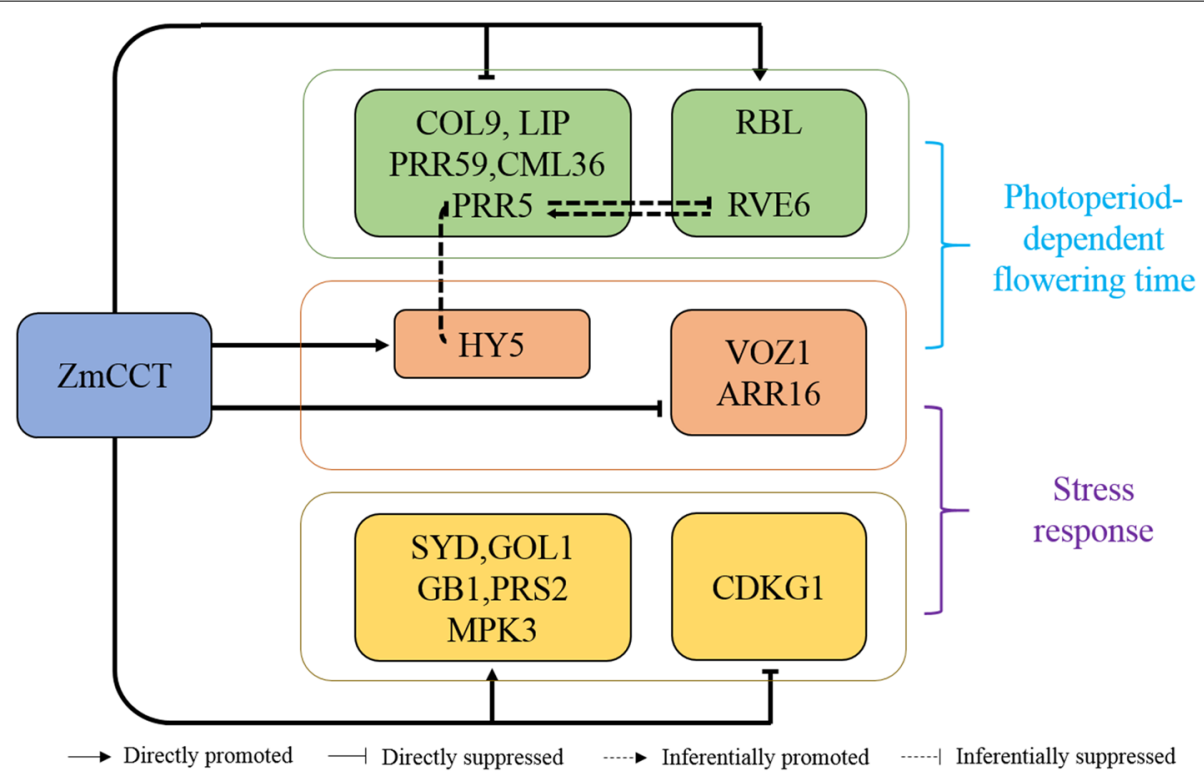

Fig. 7 A regulatory model of the roles of $Z m C C T$ gene in regulating flowering-time and stresses response in maize. T-bars indicate suppression. Dashed t-bars indicate inferentially suppressed. Arrows indicate positive regulation. Dashed arrows indicate inferentially promoted. Genes in the green boxes represent the target genes involved in the photoperiod-dependent flowering regulation. Genes in the yellow boxes represent the target genes involved in the stress response. Genes in the orange boxes represent the target genes that may have dual functions in regulating flowering time and response to stress

compared to the WT. Secondly, out of the identified 48 DEGs related to stress response, 40 genes were strongly upregulated in the transgenic plants overexpressing the ZmCCT compared to the WT.

\section{Conclusions}

$Z m C C T$ has dual functions in regulating maize flowering time and stress response. $Z m C C T$ negatively regulates flowering time and enhances maize drought tolerance under LD conditions. ZmCCT delays flowering by repressing flowering time-related genes and enhances stress tolerance by promoting the stress-responsive genes.. Based on the results of this study, a model for the regulatory role of $\mathrm{ZmCCT}$ in flowering time and stress response in maize was proposed. Our results contribute to a comprehensive understanding of the regulatory mechanisms of $\mathrm{ZmCCT}$ in regulating flowering time and stress response in maize.

\section{Methods}

\section{Plant materials and growth conditions}

In this study, the full-length coding sequence of the $Z m C C T$ was inserted into pCAMBIA1300-35S binary vector to overexpress $Z m C C T$. The wild-type (WT) B104 and $Z m C C T$ overexpression transgenic (OE-ZmCCT) plants with B104 background were used. The B104 and
Maize genetic transformation were performed by Beijing bomeixingao Technology Company. Seeds of the WT and OE-ZmCCT plants provided by Beijing bomeixingao Technology Company. The OE-ZmCCT and WT plants were grown in growth chambers (GR64, Conviron, Canada) under either long days (LD) and short days (SD) conditions. For LD conditions $15 \mathrm{~h}$ light $/ 9 \mathrm{~h}$ dark, day temperature $28^{\circ} \mathrm{C}$, night temperature $22^{\circ} \mathrm{C}$, with a $40 \%$ relative humidity, a light intensity of $105 \mu \mathrm{mol} \mathrm{m}^{-2} \mathrm{~s}^{-1}$ in Zhengzhou, China, in the spring of 2018 were implemented. Meanwhile, under SD conditions, plants were subjected to $9 \mathrm{~h}$ light $/ 15 \mathrm{~h}$ dark, other parameters were the same as for LD conditions. In this study, leaf blade tissue was collected from the leaves of the OE-ZmCCT or WT plants grown either under LD or SD conditions at the V6 growth stage. Each sample was collected from four different randomly selected plants. Three biological replicates were collected at the same time.

\section{RNA extraction and RNA sequencing (RNA-seq)}

For each sample, total RNA was isolated using the RNeasy Plant Mini Kit (Qiagen), and then purified by magnetic stand (Invitrogen). The cDNA libraries were prepared using $5 \mu \mathrm{g}$ of the total RNA following the Illumina standard protocol (TruSeq Standard RNA LT Guide). Twelve separate cDNA libraries were constructed. An Agilent 2100 Bioanalyzer system was employed to perform the 
quality control checks of all libraries. Qualified cDNA libraries were sequenced using the Illumina HiSeq 4000 system, and $150 \mathrm{bp}$ paired-reads were generated.

\section{RNA-Seq data analysis}

The RNA-Seq data analysis was performed according to the method described by Cao et al. [67]. To validate the differences in the expression levels observed by RNA-Seq between the WT and OE-ZmCCT plants under LD and SD conditions, we performed RT-qPCR on 20 differentially expressed genes. The primer sequences used in the RT-qPCR assay are listed in Additional file 2. All analyses were conducted with three technical and biological replicates.

\section{Transcriptional activation assay in yeast}

To analyze the transcriptional activity of $Z m C C T$, the yeast strain AH109 (Stratagene, USA) that contains the lacZ and HIS3 reporter genes was used. The coding sequence (CDS) of $Z m C C T$ cloned from the maize inbred line CML288 was inserted into the pBD-GAL4 vector via $B a m H I$ and NdeI restriction enzymes to produce ZmCCT-GAL4 fusion protein. The negative control pBD-GAL4, positive control pGAL4 and pBD-ZmCCT plasmids were transfected into the AH109 cells. The transfected yeast cells were transferred to YPDA or SD/Trp/-His medium and cultured at $30^{\circ} \mathrm{C}$ for $3-5$ days. The $\beta$-Galactosidase filter assay was performed to determine the $\beta$-galactosidase activity of the transfected yeast cells (PT3024-1).

\section{Gal4/UAS system assay}

The reporter (UAS-GUS) and effector (VP16 and Gal4) constructs were previously described by Tiwari et al. [68]. The ZmCCT-GAL4 effector construct contains the full-length coding sequence of $Z m C C T$ fused into the $\mathrm{N}$-terminus of the Gal4 DNA-binding domain under the control of CaMV-35S promoter. To normalize the expression of the GUS reporter gene, the 35S-LUC construct was co-transformed as an internal control. GUS and firefly luciferase (LUC) enzymatic assays were carried out in the $N$. benthamiana leaves. Five days after subculture, protoplasts were isolated from the cells. Digestion of cell walls was performed in a solution containing $1 \%(\mathrm{w} / \mathrm{v})$ cellulase Onozuka R-10 (Serva), 0.1\% (w/v) pectinase (Sigma), 0.5\% (w/v) Macerozyme RS (Serva), and 0.25 M mannitol for $2 \mathrm{~h}$ at room temperature. The polyethylene glycol (PEG) approach was employed to transform the isolated protoplasts with $20 \mu \mathrm{g}$ DNA of the reporter and the effector constructs or the mock. The LUC substrate (Promega, Madison, WI) was prepared according to the manufacturer's instructions. An aliquot of $10 \mu \mathrm{l}$ of sample extract was mixed with $50 \mu \mathrm{l}$ of the LUC substrate, and the Zylux FB15 luminometer (Fisher Scientific, Pittsburgh, PA) was then employed to measure the luciferase activity. The fluorometry was implemented to determine the GUS activities using 4-methylumbelliferyl glucuronide as a substrate. The activity of the reporter gene was represented as the ratio of GUS to luciferase activity. The data represent the average of three biological replicates.

\section{DNA affinity purification sequencing (DAP-Seq) experiments}

DAP-Seq assay was carried out as described by O'Malley et al. [69]. The NEB Next ${ }^{\circledR}$ DNA Library Prep Master Mix set for Illumina kit (NEB \#E6040S) was implemented to prepare the DAP-Seq gDNA library. The pFN19K Vector (cat\#G184A, Promega) was employed to fuse the $\mathrm{ZmCCT}$ into the HaloTag. The TNT SP6 High-Yield Wheat Germ Protein Expression System (L3260, Promega) was then implemented to express the ZmCCT-HaloTag fusion protein. Magen HaloTag Beads (G7281, Promega) was used to purify the fusion protein. The ZmCCT-HaloTag fusion protein and $500 \mathrm{ng}$ of library DNA were co-incubated in $40 \mu \mathrm{l}$ PBS buffer with a slow shaking for $1.5 \mathrm{~h}$ in a cold room. The beads were five-times washed in $200 \mu \mathrm{l}$ PBS + NP40 (0.005\%). The supernatant was discarded and an aliquot of $25 \mu$ l of elution buffer was added. Finally, beads were incubated at $98^{\circ} \mathrm{C}$ for $10 \mathrm{~min}$ to elute DNA fragments. According to the fragment size of the library, the DAP-Seq library concentration for a given read count was measured. The mock DAP-Seq libraries used as a negative control were prepared as previously described except for adding protein to the beads.

\section{DAP-Seq data analysis}

The clean reads were aligned to the maize_V4 reference genome using the software Bowtie 2 version 2.3.4.3 at the default parameters [70]. The MACS2 algorithm (ModelBased Analysis of ChIP-Seq) version 2.2.7.1 was implemented to identify peaks with BAMPE mode [71]. Genes that contain peaks located within $3 \mathrm{~kb}$ upstream the TSS (transcription start site) or downstream TTS (transcription termination site) were defined as target genes of the $\mathrm{ZmCCT}$.

\section{Gene ontology (GO) enrichment analysis}

GO enrichment analysis was performed using the AgriGO analysis toolkit version 2.0 [28]. According to maize GAMER, the GO annotations of maize B73_V4 protein-coding genes were used as a reference [72]. The type of GO in agriGO v2.0 was set as Plant GO slim. Fisher's exact test was employed to determine the significance levels. The BY procedure in the agriGO v2.0 toolkit was implemented for multiple test corrections 
[73]. Results exhibited an FDR $\leq 0.05$ were considered as significantly enriched GO terms.

\section{Electrophoretic mobility shift assay (EMSA)}

The probes used for EMSA were labeled and annealed under the guidance of DIG Gel Shift Kit standard procedure (Roche). All binding reaction components were thawed on ice. For a binding reaction of $20 \mu \mathrm{l}, 4 \mu \mathrm{l}$ of binding buffer, $1 \mu \mathrm{l}$ of poly [d (I-C)] $(1 \mu \mathrm{g} / \mu \mathrm{l}), 1 \mu \mathrm{l}$ of poly L-lysine $(0.1 \mu \mathrm{g} / \mu \mathrm{l}), 2 \mu \mathrm{l}$ labeled probe $(0.4 \mathrm{ng} /$ $\mu \mathrm{l}), 1 \mu \mathrm{l}$ purified $\mathrm{ZmCCT}$ fusion protein $((25-75 \mathrm{ng} /$ $\mu \mathrm{l})$ and double distilled water were added. After mixing the mixture carefully, it was incubated for $15 \mathrm{~min}$ at room temperature. A native polyacrylamide gel of $6-8 \%$ acrylamide in $0.5 \times$ TBE buffer was prepared, and the binding reaction was loaded onto the gel and run in $10 \mathrm{~cm} \times 10 \mathrm{~cm} \times 0.1 \mathrm{~cm}$ PAGE at $80 \mathrm{~V}$. After electrophoresis, an equilibrated nylon membrane was carefully placed onto the gel for $30 \mathrm{~min}$ at $400 \mathrm{~mA}$ to transfer the binding reaction. The membrane was then placed on a development folder or hybridization bag and $1 \mathrm{ml}$ CSPD working solution was applied. After incubation at $37^{\circ} \mathrm{C}$ for $10 \mathrm{~min}$ to enhance the luminescent reaction, images were taken.

\section{Transient assays for in vivo activation activity}

For the dual-luciferase (Dual-LUC) assay, a fragment of about $2500 \mathrm{bp}$ of the promoter region of the potential target gene was cloned into pGreenII0800-LUC to develop Pro::LUC reporter. The full-length coding sequence of the ZmCCT was inserted into pCAMBIA1300-35S binary vector to generate the $35 \mathrm{~S}:: Z m C C T$ effector. The Dual-LUC assay was carried out in $N$. benthamiana leaves. After injection, plants were grown at $25^{\circ} \mathrm{C}$ with a $14 \mathrm{~h} / 10 \mathrm{~h}$ light/dark cycle. The protein was extracted $48 \mathrm{~h}$ after injection (Cat\# E1910, Promega). The GloMax $^{\circledR} 20 / 20$ Luminometer (Cat\# E5311, Promega) was used to measure the LUC activity. An aliquot of $100 \mu \mathrm{l}$ of Stop and Glow Buffer was then added to the reaction before measuring the Renilla luciferase (REN) activity. Each sample has three biological replicates.

\section{Drought stress experiment}

For drought stress experiments, two OE-ZmCCT lines were used. The OE-ZmCCT and WT plants were grown for 2 weeks in pots filled with garden soil under LD conditions. A well-irrigated (WI) and drought-stressed (DS) trials were conducted. The WI trial was irrigated with about $500 \mathrm{ml}$ water per pot each day. The droughtstressed (DS) trial was also irrigated as the WI trial for 14 days, and then irrigation has been withheld [74]. The relative water contents (RWC) were measured in the OE-ZmCCT and WT plants to identify phenotypic variations. The RWC was calculated as essentially described in
Kwasniewski et al. [75]. Leaves from the OE-ZmCCT and WT plants were sampled for RNA extraction. Each sample was collected from three different randomly selected plants. Data shown as an average of three biological replicates.

\section{Real-time reverse transcription PCR (RT-qPCR)}

Total RNA was extracted using PureLink ${ }^{\mathrm{TM}}$ RNA Mini Kit (12183018A, ThermoFisher). An aliquot of $1.5 \mu \mathrm{g}$ RNA was reverse transcribed using the Hifair ${ }^{\circledR}$ III 1st Strand cDNA Synthesis SuperMix for RT-qPCR (YEASEN). following the manufacturer's procedure. RT-qPCR was performed using the SYBR ${ }^{\circledR}$ Green PCR Master Mix (ThermoFisher) on a LightCycler ${ }^{\circledR}$ 480II Sequence Detection System. Relative gene expression was measured according to the $2-\Delta \Delta \mathrm{Ct}$ approach. The $18 \mathrm{~S}$ ribosomal gene was selected to normalize gene expression for RT-qPCR. The primer sequences used in the RT-qPCR assay are listed in Additional file 2.

\section{Abbreviations}

DAP-Seq: DNA affinity purification sequencing; EMSA: The electrophoretic mobility shift assay; GO: Gene Ontology; LUC: Firefly luciferase; Dual-Luc: Dualluciferase transient transcriptional activity assay; RWC: Relative water content.

\section{Supplementary Information}

The online version contains supplementary material available at https://doi. org/10.1186/s12870-021-03231-y.

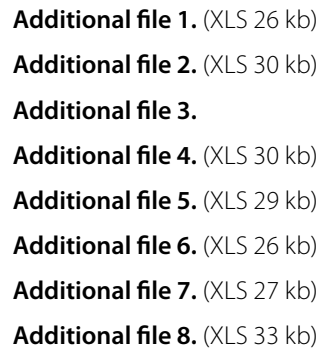

Acknowledgements

Not applicable.

\section{Authors' contributions}

L.K. and Y.C. designed the research; H.S., J.L., and H.C. analyzed data; H.S., J.L., H.C., D.D., Z.R., J.X., Z.C., and F.G. performed experiments; H.S. and S.F.A. involved in drafting the manuscript; L.K., H.S., and S.F.A. involved in revising the manuscript; H.S., J.L., and S.F.A. contributed equally to this study. All authors read and approved the final manuscript.

\section{Authors' information}

Not applicable.

\section{Funding}

This work, the library construction of RNA-Seq and DAP-Seq, the high throughput sequencing was supported by the National Natural Science Foundation of China (No.U2004158) and National Key Research and Development Program of China (2016YFD0101803). The funders had no role in the design of the study and collection, analysis, and interpretation of data and in writing the manuscript. 


\section{Availability of data and materials}

The raw data can be accessed from the NCBI Sequence Read Archive (SRA) platform under the accession number PRJNA727729 (http://www.ncbi.nlm. nih.gov/bioproject/727729).

\section{Declarations}

\section{Ethics approval and consent to participate}

The research on plants, including the collection of plant material complies with relevant institutional, national, and international guidelines and legislation.

Experimental research and field studies on plants were carried out by Huihui Su, Jiachen Liang and Haiyang Cheng.

The inbred of maize B104 and ZmCCT overexpression transgenic (OE-ZmCCT ) plants with B104 background were used in our experiment which were collected from Beijing bomeixingao Technology Company.

\section{Consent for publication}

Not applicable.

\section{Competing interests}

The authors declare that they have no competing interests.

\section{Author details}

${ }^{1}$ Synergetic Innovation Center of Henan Grain Crops and National Key Laboratory of Wheat and Maize Crop Science, Henan Agricultural University, Zhengzhou 450046, Henan, China. ${ }^{2}$ Agronomy Department, Faculty of Agriculture, Assiut University, Assiut, Egypt.

Received: 3 May 2021 Accepted: 23 September 2021

Published online: 06 October 2021

\section{References}

1. Matsuoka Y, Vigouroux Y, Goodman MM, Sanchez GJ, Buckler E, Doebley $\mathrm{J}$ : A single domestication for maize shown by multilocus microsatellite genotyping. Proc Natl Acad Sci 2002, 99(9):6080-6084.

2. Abou-Elwafa SF, Büttner B, Chia T, Schulze-Buxloh G, Hohmann U, MutasaGöttgens $E$, et al. Conservation and divergence of autonomous pathway genes in the flowering regulatory network of Beta vulgaris. J Exp Bot. 2011;62(10):3359-74.

3. Büttner B, Abou-Elwafa SF, Zhang W, Jung C, Müller AE. A survey of EMS-induced biennial Beta vulgaris mutants reveals a novel bolting locus which is unlinked to the bolting gene B. Theor Appl Genet. 2010;121(6):1117-31.

4. Jin M, Liu X, Jia W, Liu H, Li W, Peng Y, et al. ZmCOL3, a CCT gene represses flowering in maize by interfering with the circadian clock and activating expression of ZmCCT. J Integr Plant Biol. 2018;60(6):465-80.

5. Hung H-Y, Shannon LM, Tian F, Bradbury PJ, Chen C, Flint-Garcia SA, et al. ZmCCT and the genetic basis of day-length adaptation underlying the postdomestication spread of maize. Proc Natl Acad Sci. 2012;109(28):E1913-21.

6. Yang Q, Li Z, Li W, Ku L, Wang C, Ye J, et al. CACTA-like transposable element in ZmCCT attenuated photoperiod sensitivity and accelerated the postdomestication spread of maize. Proc Natl Acad Sci. 2013:110(42):16969-74

7. Huang C, Sun H, Xu D, Chen Q, Liang Y, Wang X, et al. ZmCCT9 enhances maize adaptation to higher latitudes. Proc Natl Acad Sci U S A 2018;115(2):E334-e341.

8. Guo L, Wang X, Zhao M, Huang C, Li C, Li D, et al. Stepwise cis-Regulatory Changes in ZCN8 Contribute to Maize Flowering-Time Adaptation. Current Biology. 2018;28(18):3005-15 e3004.

9. Meng X, Muszynski MG, Danilevskaya ON. The FT-like ZCN8 gene functions as a floral activator and is involved in photoperiod sensitivity in maize. Plant Cell. 2011;23(3):942-60.

10. Liang Y, Liu Q, Wang X, Huang C, Xu G, Hey S, et al. ZmMADS69 functions as a flowering activator through the ZmRap2.7-ZCN8 regulatory module and contributes to maize flowering time adaptation. New Phytol. 2019:221(4):2335-47.
11. Danilevskaya ON, Meng X, Selinger DA, Deschamps S, Hermon P, Vansant $\mathrm{G}$, et al. Involvement of the MADS-box gene ZMM4 in floral induction and inflorescence development in maize. Plant Physiol. 2008;147(4):2054-69.

12. Yanovsky MJ, Kay SA. Living by the calendar: how plants know when to flower. Nat Rev Mol Cell Biol. 2003;4(4):265-76.

13. Corbesier $L$, Coupland G. Photoperiodic flowering of Arabidopsis: integrating genetic and physiological approaches to characterization of the floral stimulus. Plant Cell Environ. 2005;28(1):54-66.

14. Wang $X$, Wu L, Zhang S, Wu L, Ku L, Wei X, et al. Robust expression and association of ZmCCA1 with circadian rhythms in maize. Plant Cell Rep. 2011;30(7):1261-72.

15. Shi Y, Zhao X, Guo S, Dong S, Wen Y, Han Z, et al. ZmCCA1a on chromosome 10 of maize delays flowering of Arabidopsis thaliana. Front Plant Sci. 2020;11:78.

16. Kumimoto RW, Zhang Y, Siefers N, Holt BF 3rd. NF-YC3, NF-YC4 and NF-YC9 are required for CONSTANS-mediated, photoperiod-dependent flowering in Arabidopsis thaliana. Plant J. 2010;63(3):379-91.

17. Cao S, Kumimoto RW, Gnesutta N, Calogero AM, Mantovani R, Holt BF 3rd. A distal CCAAT/NUCLEAR FACTOR Y complex promotes chromatin looping at the FLOWERING LOCUS T promoter and regulates the timing of flowering in Arabidopsis. Plant Cell. 2014;26(3):1009-17.

18. Hwang YH, Kim SK, Lee KC, Chung YS, Lee JH, Kim JK. Functional conservation of rice OsNF-YB/YC and Arabidopsis AtNF-YB/YC proteins in the regulation of flowering time. Plant Cell Rep. 2016;35(4):857-65.

19. Su H, Cao Y, Ku L, Yao W, Cao Y, Ren Z, et al. Dual functions of ZmNF-YA3 in photoperiod-dependent flowering and abiotic stress responses in maize. J Exp Bot. 2018;69(21):5177-89.

20. Tian L, Zhao X, Liu H, Ku L, Wang S, Han Z, et al. Alternative splicing of ZmCCA1 mediates drought response in tropical maize. PLoS One. 2019;14(1):e0211623.

21. Liu T, Carlsson J, Takeuchi T, Newton L, Farré EM. Direct regulation of abiotic responses by the Arabidopsis circadian clock component PRR7. Plant J. 2013;76(1):101-14

22. Wang W, Barnaby JY, Tada Y, Li H, Tör M, Caldelari D. Lee D-u, Fu X-D, Dong $X$ : timing of plant immune responses by a central circadian regulator. Nature. 2011:470(7332):110-4.

23. Min JH, Chung JS, Lee KH, Kim CS. The CONSTANS-like 4 transcription factor, AtCOL4, positively regulates abiotic stress tolerance through an abscisic acid-dependent manner in Arabidopsis. J Integr Plant Biol. 2015;57(3):313-24.

24. Xue $W$, Xing $Y$, Weng $X$, Zhao $Y$, Tang $W$, Wang $L$, et al. Natural variation in Ghd7 is an important regulator of heading date and yield potential in rice. Nat Genet. 2008;40(6):761-7.

25. Ku L, Tian L, Su H, Wang C, Wang X, Wu L, et al. Dual functions of the ZmCCT-associated quantitative trait locus in flowering and stress responses under long-day conditions. BMC Plant Biol. 2016;16(1):239.

26. Chettoor AM, Givan SA, Cole RA, Coker CT, Unger-Wallace E, Vejlupkova $Z$, et al. Discovery of novel transcripts and gametophytic functions via RNA-seq analysis of maize gametophytic transcriptomes. Genome Biol. 2014;15(7):414.

27. Wang B, Tseng E, Regulski M, Clark TA, Hon T, Jiao Y, et al. Unveiling the complexity of the maize transcriptome by single-molecule long-read sequencing. Nat Commun. 2016;7(1):11708.

28. Tian T, Liu Y, Yan H, You Q, Yi X, Du Z, et al. agriGO v2.0: a GO analysis toolkit for the agricultural community, 2017 update. Nucleic Acids Res. 2017;45(W1):W122-w129.

29. Gendron JM, Pruneda-Paz JL, Doherty CJ, Gross AM, Kang SE, Kay SA. Arabidopsis circadian clock protein, TOC1, is a DNA-binding transcription factor. Proc Natl Acad Sci. 2012;109(8):3167-72.

30. Song K, Kim HC, Shin S, Kim KH, Moon JC, Kim JY, et al. Transcriptome analysis of flowering time genes under drought stress in maize leaves. Front Plant Sci. 2017:8:267.

31. Wang J, Ding H, Zhang A, Ma F, Cao J, Jiang M. A novel mitogenactivated protein kinase gene in maize (Zea mays), ZmMPK3, is involved in response to diverse environmental cues. J Integr Plant Biol. 2010;52(5):442-52.

32. Wang B, Liu C, Zhang D, He C, Zhang J, Li Z. Effects of maize organ-specific drought stress response on yields from transcriptome analysis. BMC Plant Biol. 2019;19(1):335.

33. Nakai $Y$, Nakahira $Y$, Sumida H, Takebayashi K, Nagasawa $Y$, Yamasaki K et al. Vascular plant one-zinc-finger protein 1/2 transcription factors 
regulate abiotic and biotic stress responses in Arabidopsis. Plant J. 2013;73(5):761-75.

34. Cordenonsi M, Zanconato F, Azzolin L, Forcato M, Rosato A, Frasson C, et al. The hippo transducer TAZ confers cancer stem cell-related traits on breast cancer cells. Cell. 2011;147(4):759-72.

35. Overholtzer M, Zhang J, Smolen GA, Muir B, Li W, Sgroi DC, et al. Transforming properties of YAP, a candidate oncogene on the chromosome 11 q22 amplicon. Proc Natl Acad Sci U S A. 2006;103(33):12405-10.

36. Kim M, Kim T, Johnson RL, Lim DS. Transcriptional co-repressor function of the hippo pathway transducers YAP and TAZ. Cell Rep. 2015;11(2):270-82.

37. Lai X, Yan L, Lu Y, Schnable JC. Largely unlinked gene sets targeted by selection for domestication syndrome phenotypes in maize and sorghum. Plant J. 2018;93(5):843-55.

38. Du X, Wang X, Zhang J, Zhen S, Liu Y, Fu J, et al. Transcriptome analysis of leafy near-isogenic lines provides molecular insights into floral transition in maize (Zea mays). Plant Breed. 2020;139(5):883-91.

39. Lai X, Bendix C, Yan L, Zhang Y, Schnable JC, Harmon FG. Interspecific analysis of diurnal gene regulation in panicoid grasses identifies known and novel regulatory motifs. BMC Genomics. 2020;21(1):428.

40. Rawat R, Takahashi N, Hsu PY, Jones MA, Schwartz J, Salemi MR, et al. REVEILLE8 and PSEUDO-REPONSE REGULATOR5 form a negative feedback loop within the Arabidopsis circadian clock. PLoS Genet. 2011;7(3):e1001350

41. Hsu PY, Devisetty UK, Harmer SL. Accurate timekeeping is controlled by a cycling activator in Arabidopsis. eLife. 2013;2:e00473.

42. Song N, Xu Z, Wang J, Qin Q, Jiang H, Si W, et al. Genome-wide analysis of maize CONSTANS-LIKE gene family and expression profiling under light/ dark and abscisic acid treatment. Gene. 2018;673:1-11.

43. Minow MAA, Ávila LM, Turner K, Ponzoni E, Mascheretti I, Dussault FM, et al. Distinct gene networks modulate floral induction of autonomous maize and photoperiod-dependent teosinte. J Exp Bot. 2018;69(12):2937-52.

44. Cheng XF, Wang ZY. Overexpression of COL9, a CONSTANS-LIKE gene, delays flowering by reducing expression of $\mathrm{CO}$ and $\mathrm{FT}$ in Arabidopsis thaliana. Plant J. 2005:43(5):758-68.

45. Wu W, Zheng XM, Lu G, Zhong Z, Gao H, Chen L, et al. Association of functional nucleotide polymorphisms at DTH2 with the northward expansion of rice cultivation in Asia. Proc Natl Acad Sci U S A. 2013;110(8):2775-80.

46. Bendix C, Marshall CM, Harmon FG. Circadian clock genes universally control key agricultural traits. Mol Plant. 2015;8(8):1135-52.

47. Farré EM, Liu T. The PRR family of transcriptional regulators reflects the complexity and evolution of plant circadian clocks. Curr Opin Plant Biol. 2013;16(5):621-9.

48. Hajdu A, Dobos O, Domijan M, Bálint B, Nagy I, Nagy F, et al. ELONGATED HYPOCOTYL 5 mediates blue light signalling to the Arabidopsis circadian clock. Plant J. 2018;96(6):1242-54.

49. Salomé PA, To JP, Kieber JJ, McClung CR. Arabidopsis response regulators ARR3 and ARR4 play cytokinin-independent roles in the control of circadian period. Plant Cell. 2006;18(1):55-69.

50. Martí Ruiz MC, Hubbard KE, Gardner MJ, Jung HJ, Aubry S, Hotta CT, et al. Circadian oscillations of cytosolic free calcium regulate the Arabidopsis circadian clock. Nature plants. 2018:4(9):690-8.

51. Kevei E, Gyula P, Fehér B, Tóth R, Viczián A, Kircher S, et al. Arabidopsis thaliana circadian clock is regulated by the small GTPase LIP1. Curr Biol. 2007;17(17):1456-64.

52. Li A, Li G, Zhao Y, Meng Z, Zhao M, Li C, et al. Combined small RNA and gene expression analysis revealed roles of miRNAs in maize response to rice black-streaked dwarf virus infection. Sci Rep. 2018;8(1):13502.

53. Shi F, Zhang Y, Wang K, Meng Q, Liu X, Ma L, et al. Expression profile analysis of maize in response to Setosphaeria turcica. Gene. 2018;659:100-8.

54. Yu Y, Shi J, Li X, Liu J, Geng Q, Shi H, et al. Transcriptome analysis reveals the molecular mechanisms of the defense response to gray leaf spot disease in maize. BMC Genomics. 2018;19(1):742.

55. Walley JW, Shen Z, McReynolds MR, Schmelz EA, Briggs SP. Fungalinduced protein hyperacetylation in maize identified by acetylome profiling. Proc Natl Acad Sci U S A. 2018;115(1):210-5.
56. Llorente F, Alonso-Blanco C, Sánchez-Rodriguez C, Jorda L, Molina A. ERECTA receptor-like kinase and heterotrimeric $G$ protein from Arabidopsis are required for resistance to the necrotrophic fungus Plectosphaerella cucumerina. Plant J. 2005;43(2):165-80.

57. Yu GL, Katagiri F, Ausubel FM. Arabidopsis mutations at the RPS2 locus result in loss of resistance to pseudomonas syringae strains expressing the avirulence gene avrRpt2. Mol Plant-Microbe Interactions. 1993;6(4):434-43.

58. Kunkel BN, Bent AF, Dahlbeck D, Innes RW, Staskawicz BJ. RPS2, an Arabidopsis disease resistance locus specifying recognition of pseudomonas syringae strains expressing the avirulence gene avrRpt2. Plant Cell. 1993:5(8):865-75.

59. Rojas CM, Senthil-Kumar M, Wang K, Ryu CM, Kaundal A, Mysore KS. Glycolate oxidase modulates reactive oxygen species-mediated signal transduction during nonhost resistance in Nicotiana benthamiana and Arabidopsis. Plant Cell. 2012;24(1):336-52.

60. Wu Q, Xu F, Liu L, Char SN, Ding Y, Je Bl, et al. The maize heterotrimeric $G$ protein $\beta$ subunit controls shoot meristem development and immune responses. Proc Natl Acad Sci U S A. 2020;117(3):1799-805.

61. Li H, Huang K, Du H, Wang H, Chen X, Gao S, et al. Genome-wide analysis of Gro/Tup1 family corepressors and their responses to hormones and abiotic stresses in maize. J Plant Biol. 2016;59(6):603-15.

62. Colombo L, Marziani G, Masiero S, Wittich PE, Schmidt RJ, Gorla MS, et al. BRANCHED SILKLESS mediates the transition from spikelet to floral meristem during Zea mays ear development. Plant J. 1998;16(3):355-63.

63. Xiao W, Custard KD, Brown RC, Lemmon BE, Harada JJ, Goldberg RB, et al. DNA methylation is critical for Arabidopsis embryogenesis and seed viability. Plant Cell. 2006;18(4):805-14.

64. Yamauchi T, Johzuka-Hisatomi Y, Terada R, Nakamura I, lida S. The MET1b gene encoding a maintenance DNA methyltransferase is indispensable for normal development in rice. Plant Mol Biol. 2014;85(3):219-32.

65. Xu J, Zhang HY, Xie CH, Xue HW, Dijkhuis P, Liu CM. EMBRYONIC FACTOR 1 encodes an AMP deaminase and is essential for the zygote to embryo transition in Arabidopsis. Plant J. 2005;42(5):743-56.

66. Celesnik H, Ali GS, Robison FM, Reddy AS. Arabidopsis thaliana VOZ (vascular plant one-zinc finger) transcription factors are required for proper regulation of flowering time. Biology Open. 2013;2(4):424-31.

67. Cao Y, Zeng H, Ku L, Ren Z, Han Y, Su H, et al. ZmIBH1-1 regulates plant architecture in maize. J Exp Bot. 2020;71(10):2943-55.

68. Tiwari SB, Wang XJ, Hagen G, Guilfoyle TJ. AUX/IAA proteins are active repressors, and their stability and activity are modulated by auxin. Plant Cell. 2001;13(12):2809-22.

69. O'Malley RC, Huang SC, Song L, Lewsey MG, Bartlett A, Nery JR, et al. Cistrome and Epicistrome features shape the regulatory DNA landscape. Cell. 2016;165(5):1280-92.

70. Langmead B, Salzberg SL. Fast gapped-read alignment with bowtie 2. Nat Methods. 2012;9(4):357-9.

71. Zhang Y, Liu T, Meyer CA, Eeckhoute J, Johnson DS, Bernstein BE, et al. Model-based analysis of ChIP-Seq (MACS). Genome Biol. 2008;9(9):R137.

72. Wimalanathan K, Friedberg I, Andorf CM, Lawrence-Dill CJ. Maize GO annotation-methods, evaluation, and review (maize-GAMER). Plant direct. 2018;2(4):e00052.

73. Benjamini Y, Yekutieli $D$. The control of the false discovery rate in multiple testing under dependency. Ann Stat. 2001;29(4):1165 -1188, 1124.

74. Zhang $H$, Xiang Y, He N, Liu X, Liu H, Fang L, et al. Enhanced vitamin $C$ production mediated by an ABA-induced PTP-like Nucleotidase improves plant drought tolerance in Arabidopsis and maize. Mol Plant. 2020;13(5):760-76.

75. Kwasniewski M, Daszkowska-Golec A, Janiak A, Chwialkowska K, Nowakowska U, Sablok G, et al. Transcriptome analysis reveals the role of the root hairs as environmental sensors to maintain plant functions under water-deficiency conditions. J Exp Bot. 2016;67(4):1079-94.

\section{Publisher's Note}

Springer Nature remains neutral with regard to jurisdictional claims in published maps and institutional affiliations. 